Original paper

\title{
Multicellular dosimetric chain for molecular radiotherapy exemplified with dose simulations on 3D cell spheroids
}

\author{
Vappu Reijonen ${ }^{\text {b,* }}$, Liisa K. Kanninen ${ }^{c}$, Eero Hippeläinen ${ }^{a}$, Yan-Ru Lou ${ }^{c}$, Eero Salli ${ }^{\text {a }}$, Alexey Sofiev ${ }^{\text {a,d }}$, \\ Melina Malinen ${ }^{\mathrm{c}}$, Timo Paasonen ${ }^{\mathrm{b}, \mathrm{d}}$, Marjo Yliperttula ${ }^{\mathrm{c}}$, Antti Kuronen ${ }^{\mathrm{d}}$, Sauli Savolainen ${ }^{\mathrm{a}, \mathrm{d}}$ \\ ${ }^{a}$ HUS Medical Imaging Center, Helsinki University Hospital, Finland \\ ${ }^{\mathrm{b}}$ Comprehensive Cancer Center, Helsinki University Hospital, Finland

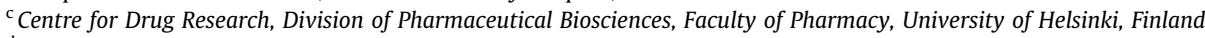 \\ ${ }^{\mathrm{d}}$ Department of Physics, University of Helsinki, Finland
}

\section{A R T I C L E I N F O}

\section{Article history:}

Received 26 June 2016

Received in Revised form 25 May 2017

Accepted 13 July 2017

Available online 20 July 2017

\section{Keywords:}

\section{Dosimetry}

Radiopharmaceuticals

Radiation dose-response relationship

Cell-level

\begin{abstract}
A B S T R A C T
Purpose: Absorbed radiation dose-response relationships are not clear in molecular radiotherapy (MRT) Here, we propose a voxel-based dose calculation system for multicellular dosimetry in MRT. We applied confocal microscope images of a spherical cell aggregate i.e. a spheroid, to examine the computation of dose distribution within a tissue from the distribution of radiopharmaceuticals.

Methods: A confocal microscope Z-stack of a human hepatocellular carcinoma HepG2 spheroid was segmented using a support-vector machine algorithm and a watershed function. Heterogeneity in activity uptake was simulated by selecting a varying amount of the cell nuclei to contain ${ }^{111} \mathrm{In},{ }^{125} \mathrm{I}$, or ${ }^{177} \mathrm{Lu}$. Absorbed dose simulations were carried out using vxlPen, a software application based on the Monte Carlo code PENELOPE.

Results: We developed a schema for radiopharmaceutical dosimetry. The schema utilizes a partially supervised segmentation method for cell-level image data together with a novel main program for voxel-based radiation dose simulations. We observed that for ${ }^{177} \mathrm{Lu}$, radiation cross-fire enabled full dose coverage even if the radiopharmaceutical had accumulated to only $60 \%$ of the spheroid cells. This effect was not found with ${ }^{111}$ In and ${ }^{125}$ I. Using these Auger/internal conversion electron emitters seemed to guarantee that only the cells with a high enough activity uptake will accumulate a lethal amount of dose, while neighboring cells are spared.

Conclusions: We computed absorbed radiation dose distributions in a 3D-cultured cell spheroid with a novel multicellular dosimetric chain. Combined with pharmacological studies in different tissue models, our cell-level dosimetric calculation method can clarify dose-response relationships for radiopharmaceuticals used in MRT.
\end{abstract}

๔ 2017 Associazione Italiana di Fisica Medica. Published by Elsevier Ltd. All rights reserved.

\section{Introduction}

Molecular radiotherapy (MRT) makes use of different cell mechanisms (e.g. receptor binding) to deliver lethal radiation doses selectively to malignant cells. Despite the large number of treated patients and successful clinical trials over the past decades, considerable uncertainties still remain regarding the optimization of MRT $[1,2]$. The dosimetry of MRT is still developing, and the key issue to establish is how the administered activity, the absorbed radiation dose distribution, and the clinical response and side effects relate

\footnotetext{
* Corresponding author at: Comprehensive Cancer Center, Helsinki University Hospital, P.O. Box 180, 00290 Helsinki, Finland.

E-mail address: vappu.reijonen@hus.fi (V. Reijonen).
}

to each other, and how this knowledge can be used to plan more individualized treatments.

For internally administered radionuclides, absorbed radiation dose calculations have long based on scintigraphy studies combined with the medical internal radiation dose (MIRD) formalism on a macroscopic level $[3,4]$. Applying the MIRD formalism on a microscopic level has also been studied [5]. The cell-scale morphology of tumors (cell dimensions and tissue packing) shows variation both between patients and within a patient. Relatively long irradiation ranges can balance effects of small-scale non-uniformities in the activity distribution. However, for short-range radiation, the absorbed radiation dose distribution can contain steep gradients in accordance with the non-homogeneous distribution of the radiopharmaceutical between cell surfaces, cytoplasm, and nuclei 
depending on the cell type. For example, in the case of peptide receptor radionuclide therapy (PRRT), it has been shown that using a different chelator, peptide and/or radionuclide can affect strongly the cellular binding affinities of the radiopharmaceutical. This in turn results in differing uptake and dose distribution in the malignant and normal tissues.

Studying internal dosimetry at biologically relevant scales appears to be essential for the development of MRT [6]. Therefore, utilizing in vivo mimicking tumor cell cultures is valuable. In vitro three-dimensionally (3D-)cultured tumor cell aggregates, also known as spheroids, resemble their in vivo counterparts more closely than conventional two-dimensional (2D) cell culture systems; the cells cultured in 3D retain their natural architecture and functionality better than those cultured in 2D. Various culturing techniques have been exploited to create 3D tumor models to study tumor biology, pathogenesis as well as the effect of anti-cancer compounds [7], including radiopharmaceuticals used in MRT [8,9].

3D cell cluster computational models have frequently been based on simple geometries like nested spheres, cylinders, and cubes mimicking cells and nuclei $[5,10]$. However, the computational capacity and imaging methods that are accessible today enable more sophisticated cell-level dosimetric modeling. A 3Dcultured tumor can provide detailed morphological data as a starting point for the MRT dosimetric chain. The cellular structures such as the nucleus, cytoskeleton, and plasma membrane can be visualized using fluorescent labels and confocal microscopy. Furthermore, the cell-level distribution of radiopharmaceuticals can be studied by labeling [11].

To perform dose calculations, the imaging data needs to be segmented. Manual cell tracking is labor-intensive and subjective, and therefore automated segmentation algorithms are needed. Intensity thresholding is frequently used in the segmentation of microscopy images [12]; however, thresholding cannot be applied to multichannel data and cells (or other structures) that touch each other and are inseparable. Watershed methods [13] are often used to segment touching objects, but they tend to lead to oversegmentation. To overcome these problems, new ways to classify and regularize image data for segmentation algorithms are needed. A support-vector machine (SVM) [14] can be efficiently taught to classify structures from 3D confocal microscope data by giving it a set of training voxels and their classification. In this study, we utilized such an SVM classifier and morphological preprocessing to avoid oversegmentation in combination with a watershed trans- form to create a segmentation process which has potential to be fully automated with more datasets.

Here, we present a new multicellular dosimetric chain (see the schematic Fig. 1) that combines confocal microscope imaging data of a 3D-cultured tumor cell spheroid, an automated segmentation algorithm, and Monte Carlo (MC) dosimetric calculations to assess the absorbed radiation dose distribution down to the cell-scale (micrometer scale). To exemplify, we simulate the behavior of activity vs. dose distributions in the case of the Auger electron and internal conversion (IC) electron emitters ${ }^{125} \mathrm{I}$ and ${ }^{111} \mathrm{In}$, and the low-energy $\beta$ electron emitter ${ }^{177} \mathrm{Lu}$, all of which have very distinct spectra of the emitted electron and photon radiation [15]. ${ }^{125} \mathrm{I}$ is a radionuclide that has been studied widely regarding multicellular dosimetry [9] and it can be considered as a benchmark ("a model Auger emitter") although its clinical applications for MRT have been limited. On the other hand, ${ }^{177} \mathrm{Lu}$ is actively used today for MRT in PRRT, and also in e.g. ${ }^{177}$ Lu-PSMA therapy. Before ${ }^{177} \mathrm{Lu}$, ${ }^{111}$ In was used for PRRT, with less efficient results; a fact that makes the comparison of these two radionuclides intriguing. Performing dose calculations with MC methods can be timeconsuming, and more effective ways, such as utilizing dose point kernels (DPKs), have often been preferred. To corroborate our MC dosimetric calculations, we also produced DPKs and compared them to published results.

\section{Materials and methods}

\subsection{Tumor spheroid culture and imaging}

Human hepatocellular carcinoma HepG2 (HB-8065, ATCC) cells [16] were maintained in $75 \mathrm{~cm}^{2}$ culture flasks in DMEM (31966021, Gibco) supplemented with $10 \%$ fetal bovine serum, $100 \mathrm{IU} /$ $\mathrm{ml}$ penicillin, and $100 \mu \mathrm{g} / \mathrm{ml}$ streptomycin. During the maintenance the cells were subcultured twice a week in a ratio of 1:5.

The cells were transferred to 3D culture environments by embedding them in GrowDex ${ }^{\circledR}$, a nanofibrillar cellulose hydrogel, as previously described by us $[17,18]$. Cultures were formed in an 8-well Chamber Slide system (177445, Nunc). Thereafter, the cells were detached with $0.25 \%$ trypsin-EDTA (25200-072, Gibco) and the cell suspension was mixed with the hydrogel and culture medium to obtain a 1.0-1.2 wt\% hydrogel concentration and a cell density of $0.8 \times 10^{6}$ cells $/ \mathrm{ml}$. Lastly, a culture medium was added
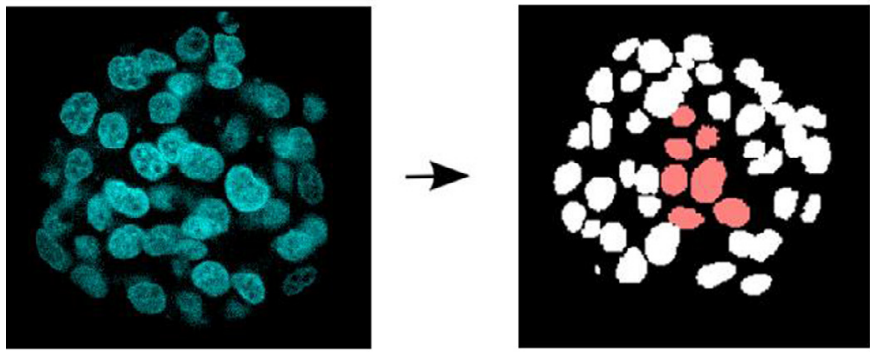

Confocal microscope imaging

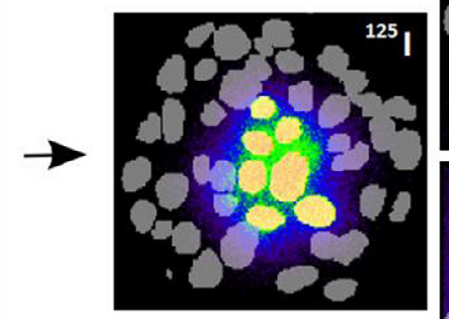

Absorbed dose simulations

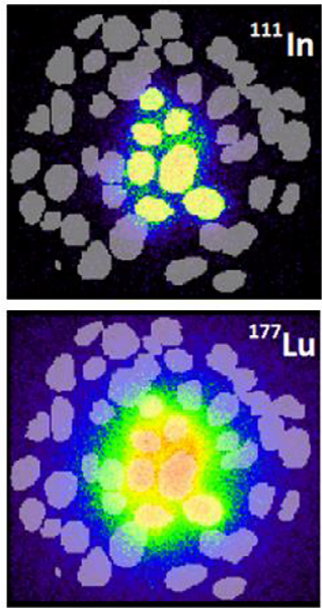

Fig. 1. Multicellular dosimetric chain: a confocal microscope image of a cell spheroid was segmented and used as a basis for dosimetric calculations. On the left, the Hoechst

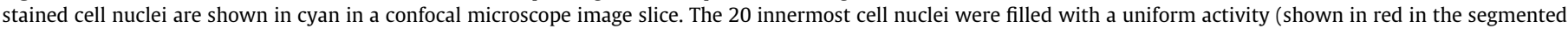
image slice in the middle), and the resulting absorbed radiation dose distributions are shown on the right (the colour scale in the images is schematic). 


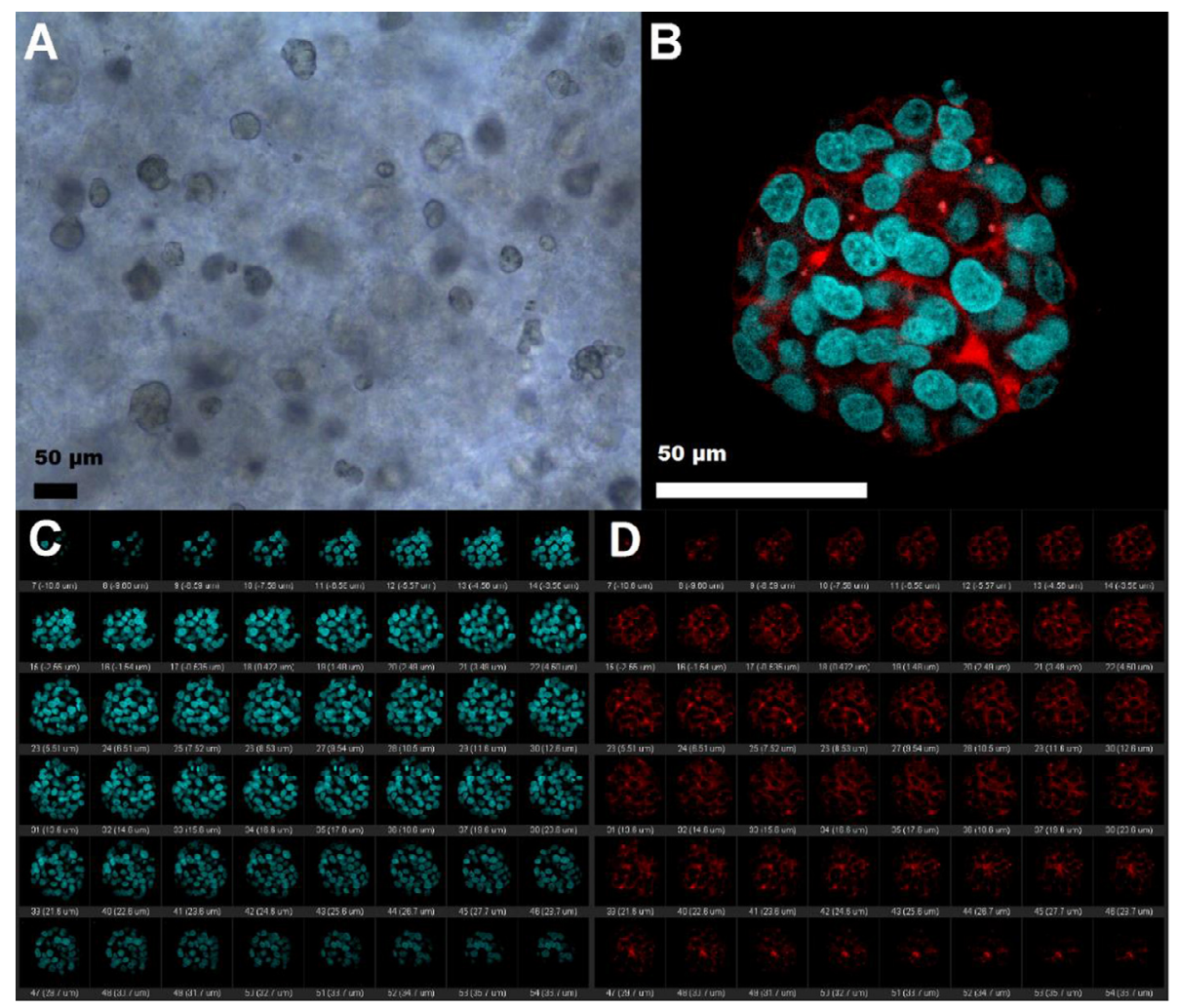

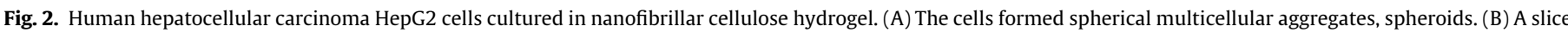

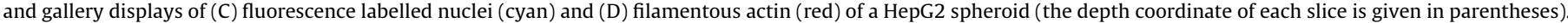
(For interpretation of the references to colour in this figure legend, the reader is referred to the web version of this article.)

on top of the hydrogel. The cell cultures were maintained at $37^{\circ} \mathrm{C}$ in a humid atmosphere with $5 \% \mathrm{CO}_{2}$.

After four days in the culture, HepG2 cells had formed 3D multicellular aggregates, spheroids. The spheroids were fixed with $3 \%$ paraformaldehyde for $15 \mathrm{~min}$ followed by $15 \mathrm{~min}$ of cell membrane permeation with $0.1 \%$ Triton-X-100 (T-8787, SigmaAldrich). Cell cytoskeleton filaments, actin filaments (F-actin), were stained with Alexa Fluor 594 Phalloidin (1:100 in 1xPBS, A12381, Invitrogen), and the nuclei were stained with $1 \mu \mathrm{g} / \mathrm{ml}$ Hoechst 33258 (bisbenzimide, Sigma-Aldrich) for $30 \mathrm{~min}$. The sample was mounted with ProLong Gold antifade reagent (P36934, Invitrogen) and analyzed with a confocal microscope (Leica TCS SP5, HCX APO 63x/1,30 Corr glycerol objective) using a UV diode $405 \mathrm{~nm} / 50 \mathrm{~mW}$ for Hoechst and a laser DPSS $561 \mathrm{~nm} / 20 \mathrm{~mW}$ for Alexa Fluor 594. To form the Z-stack, the whole depth of the spheroid was scanned with a $1 \mu \mathrm{m}$ step size (Fig. 2).

\subsection{Segmentation of cells from confocal microscope image}

For creating our dosimetric chain, we developed a new partially supervised segmentation method for 3D confocal microscope images. The method consists of a) image data processing steps, b) support-vector machine [14] based classification, and c) watershed-based [13] delineation of structures. The segmentation method was applied to segment the cell nuclei from the 3D confocal microscope image of the HepG2 spheroid represented in Fig. 2 utilizing the filamentous actin and the nucleus channels.

The preprocessing of the confocal microscope images was carried out for both the nucleus and the F-actin (cytoskeleton) staining channels in the following way: the image voxels were resampled to be isotropic using linear interpolation (to the cubical voxel size of $0.21 \mu \mathrm{m}$, which was the smallest imaging spacing), and a Gaussian filter (convolution kernel $5 \times 5 \times 5$, standard deviation 0.65 voxels) was applied to decrease noise in the image.
Next, an SVM classifier (Gaussian radial basis function kernel) was trained to classify the image voxels to either "nucleus" or "non-nucleus" based on the intensity information from the staining channels by using the fitcsvm function from the Statistics and Machine learning toolbox of Matlab (Mathworks Inc.; default parameters were used, except that standardization of the predictor was enabled). In this case, $2^{10}(1024)$ random points from the manual segmentation of the same image were used as the set of training voxels for SVM. In the resulting binary images, there were some clearly misclassified voxels (e.g. a single "non-nucleus" voxel in the middle of a group of "nucleus" voxels) and thus the classification was morphologically processed by connectivity-based hole-filling and edge smoothing by dilatation and erosion $(5 \times 5 \times 5$ neighborhood).

The next step was to perform a distance transform: each voxel was assigned a number that was the Euclidean distance between the voxel and the closest background voxel. Thereafter, the data from the staining channels and a Sobel gradient estimation for edge detection from the nucleus channel were combined with the distance transform. To avoid oversegmentation, this combination is processed using morphological image reconstruction [13] with a 6-connected neighborhood. This was translated by a watershed algorithm: the furthest points from the edges were recognized as centers of cell nuclei, and the watershed algorithm defined borderlines between them. This way, cell nuclei were identified and labeled.

\subsection{Dose calculation with the voxel geometry Monte Carlo dosimetry code vxlPen}

PENELOPE Monte Carlo code [19] (rev. 2011) was used for radiation dose simulations in the spheroid geometry. The code simulates coupled electron-photon transport in arbitrary materials 
down to a few hundred eVs. Simulation accuracy can be adjusted with the energy threshold for particle absorption $\left(E_{a b s}\right)$ and the maximum allowed track length between two consecutive interaction events $\left(s_{\max }\right)$. In this work, we simulated different activity distributions of ${ }^{111} \mathrm{In},{ }^{125} \mathrm{I}$, and ${ }^{177} \mathrm{Lu}$. We used the spectra from the Brookhaven National Laboratory database, available on www.doseinfo-radar.com [15]. The cut-off $E_{a b s}$ was set to $0.3 \mathrm{keV}$ for all primary and secondary particles (it is very unlikely that an electron with energy $<0.3 \mathrm{keV}$ will "escape" the current voxel, and thus it will be absorbed entirely within it) and $s_{\max }$ was set to $0.21 \mu \mathrm{m}$ (the size of the voxel side). On the studied scale, the deposited energy from the electrons for ${ }^{111} \mathrm{In},{ }^{125} \mathrm{I}$, and ${ }^{177} \mathrm{Lu}$ is at least about a thousand times higher than from the photons (cf. [20]), and thus the photon contribution was omitted.

In our previous work [21], we developed a new main program vxlPen for voxel-level radiation dose simulations to support extensive voxel dose tracking and voxel source geometries. Initially the code was developed for using CT and SPECT images, but it can similarly be applied to segmented cell image data and a given activity distribution. From the given activity distribution, vxlPen formed a corresponding probability distribution to generate particles (radiation) according to the given decay spectrum. The interactions of the particles with the medium were then modeled and tracked by the code, and the resulting absorbed dose distribution was saved in the voxel geometry. In this work, we used liquid water as the medium throughout the simulations. vxlPen output dose rate $[\mathrm{mGy} / \mathrm{MBq} / \mathrm{s}]$ and its standard deviation in the voxel coordinates of the segmented 3D confocal microscope images.

To illustrate the differences between the three chosen radionuclides, their electron dose point kernels (DPK) were simulated (Fig. 3). The shapes of the DPKs were as expected: the part of the ${ }^{111}$ In DPK arising from the decay electrons is narrower than the corresponding part of ${ }^{125} \mathrm{I}$, whereas the ${ }^{177} \mathrm{Lu}$ DPK extends much further due to beta emission. The DPKs agree with earlier results in the literature [20].

To demonstrate the voxel-based dosimetry calculation of our multicellular dosimetric chain, we ran vxlPen for each of the radionuclides ${ }^{111} \mathrm{In},{ }^{125} \mathrm{I}$, and ${ }^{177} \mathrm{Lu}$ so that a certain percentage, namely $5 \%, 20 \%, 40 \%, 60 \%$ and $100 \%$, of the HepG 2 spheroid cell nuclei segmented by the algorithm were filled with uniform activity and chosen at random (using a pseudo-random number gener-

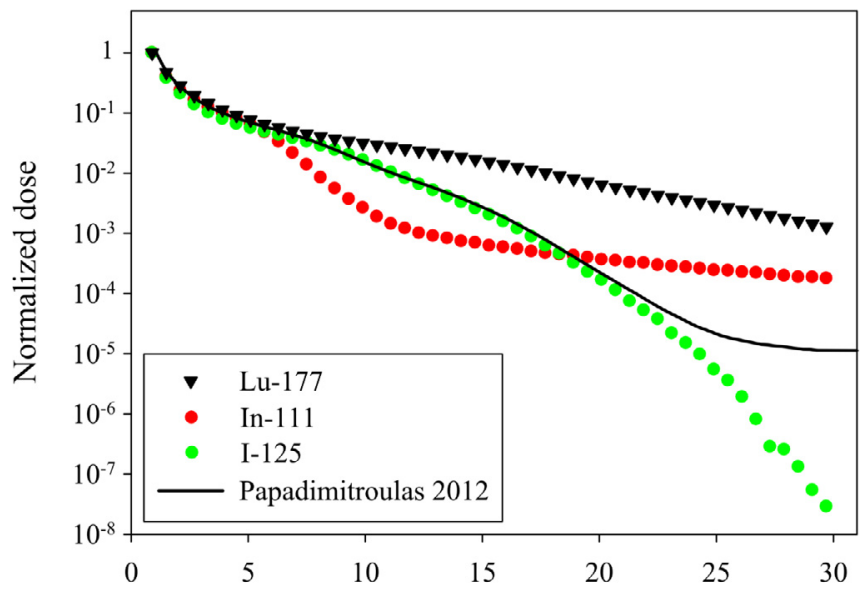

Distance from a point source $[\mu \mathrm{m}]$

Fig. 3. Simulated electron dose point kernels of ${ }^{177} \mathrm{Lu},{ }^{111} \mathrm{In}$, and ${ }^{125} \mathrm{I}$ in water. The value of the DPK at a certain distance gives the energy deposited into a spherical shell at that distance. To compare with the literature, the total DPK of ${ }^{125} \mathrm{I}$ in water calculated using the GATE v6.1 Monte Carlo toolkit in Ref. [20] is presented (solid black line). The tails of the ${ }^{125}$ I DPKs differ due to the photon contribution included in the DPK of Papamitroulas et al. ator, once for each percentage). All the voxels outside the chosen cell nuclei were left empty, regardless of whether they belonged to the background or to the other cell nuclei: that is, either all the voxels of a certain cell nucleus had the same activity or no activity at all (like the background). Different levels of activity uptake in the cells can be simulated by the code, and more complex activity distributions could be studied in the future.

\section{Results}

\subsection{Segmentation}

Manual segmentation of the cell nuclei from the volumetric confocal microscopy images of a chosen HepG2 spheroid (Fig. 4) was performed for all slices using Slicer 4.5 [22]. 200 nuclei were identified in this way. When using the segmentation algorithm described in Section 2.2, 230 nuclei were found (+15\%). Some of the nuclei outlines appear to be quite irregular in the algorithmbased segmentation.

For this work, we compared the resulting cell segmentation of the HepG2 spheroid from a few different automated segmentation methods against the manually segmented data using the Dice score [23] and the SEG accuracy measure [24]. The Dice score, also called the overlap index, measures the congruence of the segmentation produced by different methods against the ground truth (which in this case is the manual segmentation). However, the Dice score does not measure whether cells that appear fused in the image have been separated correctly. For this task, the SEG accuracy measure was used. The SEG accuracy measure, defined in Ref. [24], is the mean of the Jaccard similarity indices (i.e. intersections over unions) between the matching reference cells and the automatically segmented cells. A reference cell and a segmented cell are considered matching if their intersection is more than $50 \%$ of the reference cell. For this work, we calculated two SEG measures. Firstly, the manually delineated nuclei were defined as "the reference cells" and the nuclei segmented by the algorithm were "the segmented cells". Secondly, the nuclei segmented by the algorithm were defined as "the reference cells" and the manually segmented cells were "the segmented cells". The final SEG measure was then defined as the mean of these two SEG measures.

\subsubsection{SVM vs. intensity threshold classification}

The Dice score for Otsu's thresholding [25] using only the nucleus channel is 0.45 . The SVM classification based on the nucleus and F-acting staining channels produced a result of 0.83 .

\subsubsection{Modified watershed vs. basic watershed delineation}

Using the SVM classification, the basic Watershed method, based on the distance transform of the SVM classification, produced a SEG measure of 0.14 . The method presented in this work produced 0.59 .

Thus, expressed in a quantifiable manner, the segmentation method studied in this work led to a result that is much closer to the manual segmentation than using intensity threshold classification and basic watershed delineation.

\subsection{Dose distributions for different activity distributions}

In Fig. 5A-C, normalized absorbed doses to the HepG2 spheroid cell nuclei are shown cell by cell for the varying amount of cells containing activity $(5 \%, 20 \%, 40 \%, 60 \%$, and $100 \%$ of the 230 nuclei segmented by the algorithm). The figures show how ${ }^{177} \mathrm{Lu}$ produces vastly more cross-fire irradiation than ${ }^{111}$ In and ${ }^{125}$ I. According to our simulation, the absorbed dose distribution in the case of 


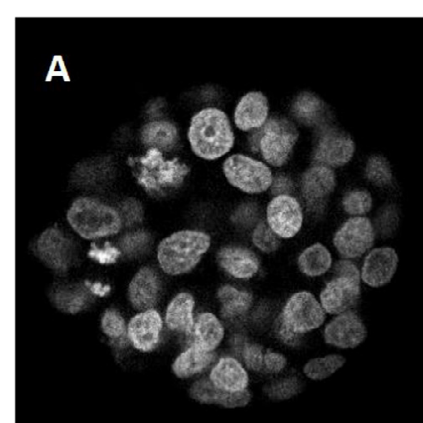

D

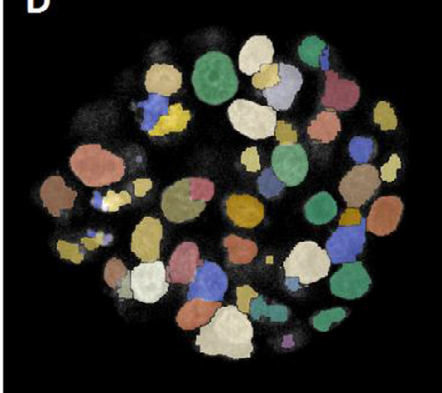

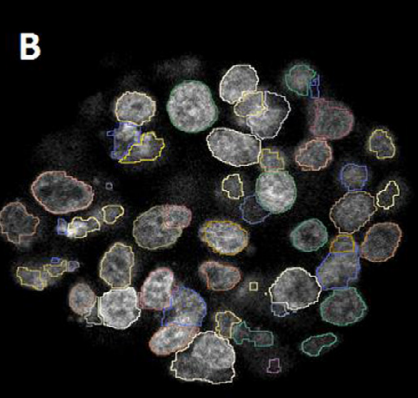

$\mathbf{E}$

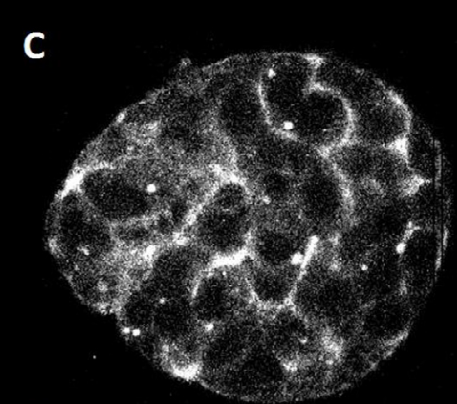

$\mathbf{F}$

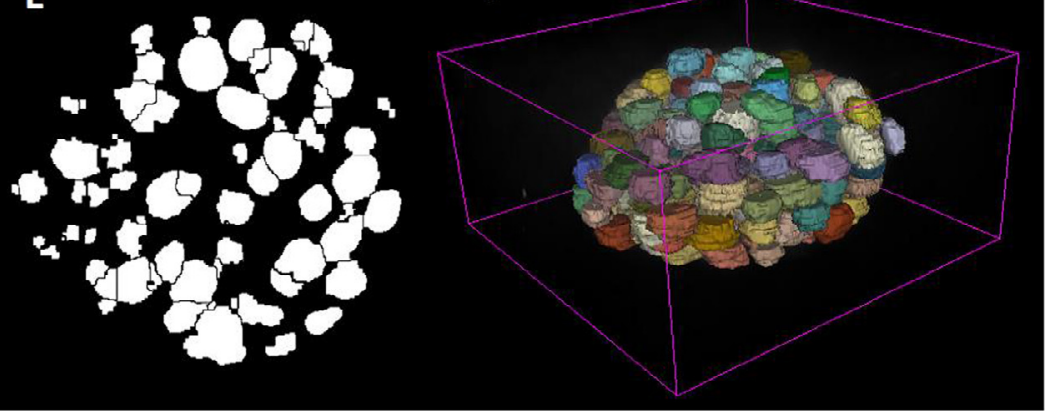

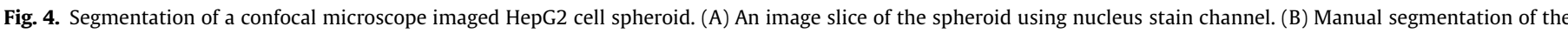

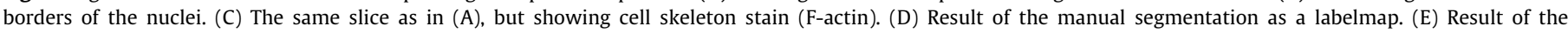
segmentation algorithm showing the nuclei in white. (F) 3D visualization of the manual segmentation.

\begin{abstract}
${ }^{177}$ Lu covers all the cells in the spheroid even with only $20 \%$ nuclei activation. Interestingly, after $60 \%$ of random nuclei activation the ${ }^{177} \mathrm{Lu}$ dose coverage over the spheroid does not significantly decrease when compared to the case where all the nuclei are activated. The cross-fire dose of ${ }^{111}$ In and ${ }^{125}$ I is comparatively diminished so that it is possible that only the cells that exhibit sufficient activity uptake can receive a lethal radiation dose, while the neighboring cells are exposed only to a fraction of the dose and are potentially spared from deterministic radiation damage. The results support our hypotheses and could also be qualitatively concluded already from the DPKs (see Fig. 2).
\end{abstract}

In Fig. 5A and $\mathrm{C}$, there are three arbitrary dose thresholds (dashed lines), i.e. $30 \%, 40 \%$ and $50 \%$ of the maximum dose. In Fig. 6, the number of cells above the threshold is shown as a function of the nuclei with activity uptake. Let us hypothesize that this dose threshold indicates a lethal cell dose: the cells that receive a higher absorbed dose do not survive. Different tumor cells are known to have different radiosensitivities (due to factors such as proliferation rate, cell cycle, etc.), which could result in the existence of such varying values for the threshold. For ${ }^{111}$ In, only the cells that were activated receive a dose that is above the chosen thresholds, and thus the plot in Fig. 6 is linear and the same for each of the threshold levels. For ${ }^{177} \mathrm{Lu}$ the behavior of the function is more complex and is dependent on the threshold value for absorbed dose: if a lower absorbed dose is enough to kill a cell, a more heterogeneous activity distribution can destroy a tumor owing to the cross-fire effect. Based on these findings, it seems that the Auger emitters ${ }^{111}$ In and ${ }^{125}$ I can destroy a tumor only when there is enough activity accumulated in each individual cell that needs to be killed. In contrast to this, the cross-fire effect of the beta emitter ${ }^{177} \mathrm{Lu}$ can counterbalance cell-level heterogeneity in the activity distribution to an extent that could be further studied by using our workflow in combination with radiobiological experiments.

When interpreting Figs. 5 and 6, it needs to be recognized that the graphs are normalized to the maximum dose achieved in each set-up. This presentation omits the yield of emitted radiation per decay. For instance, the amount of energy deposited per decay in a $5-\mathrm{nm}$ sphere is more than twice as high for ${ }^{125}$ I than for ${ }^{111}$ In (as ${ }^{125}$ I is a more prolific emitter of Auger electrons with $\sim 20$ Auger electrons per decay, whereas ${ }^{111}$ In produces $\sim 8$ Auger electrons per decay) [26], and thus more activity is needed to produce the same local dose in the case of the latter radionuclide. The representation chosen here also does not bring out the temporal dimension, including the effect of dose rate [27]. The half-lives of ${ }^{111}$ In and ${ }^{125} \mathrm{I}$ and ${ }^{177} \mathrm{Lu}$ are significantly different from each other $(2.80 \mathrm{~d}, 59.4 \mathrm{~d}$ and $6.73 \mathrm{~d}$, respectively).

\section{Discussion}

The importance of cell-level dosimetric modeling to the design of treatment plans for MRT has been understood for decades, but implementing these models in pre-clinical studies and clinical treatments has been rather slow, and tools to facilitate this are needed. Malaroda et al. [28] created a software package for multicellular dosimetry in voxel geometry, and applied it not only to a spherical model, but also to a glioma spheroid (in 2D). An interesting recent effort to create a user-friendly program, MIRDcell, for more generalizable multicellular dosimetric analysis was made by Vaziri et al. [5]. However, in MIRDcell the cell and cell nucleus are modeled as concentric spheres and imaging data cannot be fed into the program. The novelty in our multicellular dosimetric workflow is to put all the pieces of the puzzle - imaging, image processing and dose calculation - together to be applied effortlessly by the user. Many conclusions can be drawn from constructing simpler geometric 3D models; however, the power of our microscopy image-based approach is its direct and efficient applicability to any cell sample. This new approach can be applied both to pre-clinical radiopharmacological studies and to patient cell samples for individualized dosimetry.

To exemplify the workflow, we applied a 3D-cultured tumor cell aggregate, a spheroid, in absorbed radiation dose calculations to study the effect of heterogeneous activity distribution on the cell 
A.

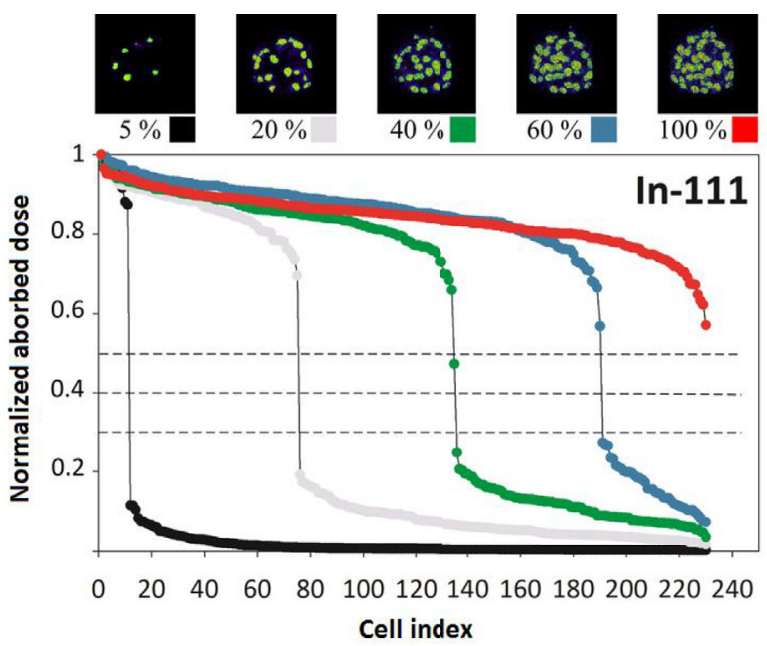

B.

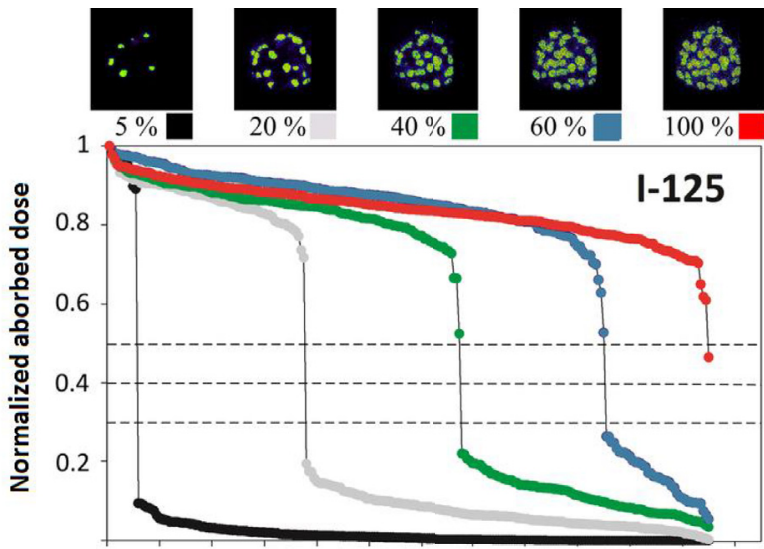

$0 \quad 20 \quad 40 \quad 60 \quad 80100120140160180200220240$

Cell index

C.

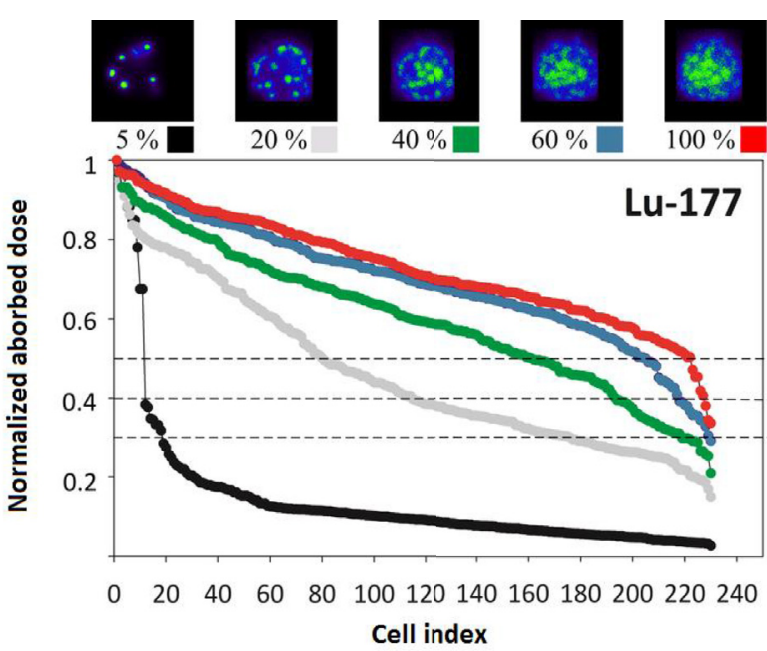

Fig. 5. (A-C) Dose distribution in the HepG2 spheroid in the case of $(A){ }^{111} \mathrm{In},(\mathrm{B}){ }^{125} \mathrm{I}$ and $(C){ }^{177} \mathrm{Lu}$, when a certain percentage of the cell nuclei is filled with activity. Doses to the cells are presented as normalized to the maximum dose reached and as a function of sorted cell index (descending dose). The dashed blue horizontal lines in (A) and (C) mark the levels of 30\%, $40 \%$ and $50 \%$ of the maximum dose, see the following Fig. 6 .

scale. Compared to human tissue samples, cultured tumor cell lines are easier to use, and widely available. Numerous tumor cell lines have been established and well-characterized, and also imple-

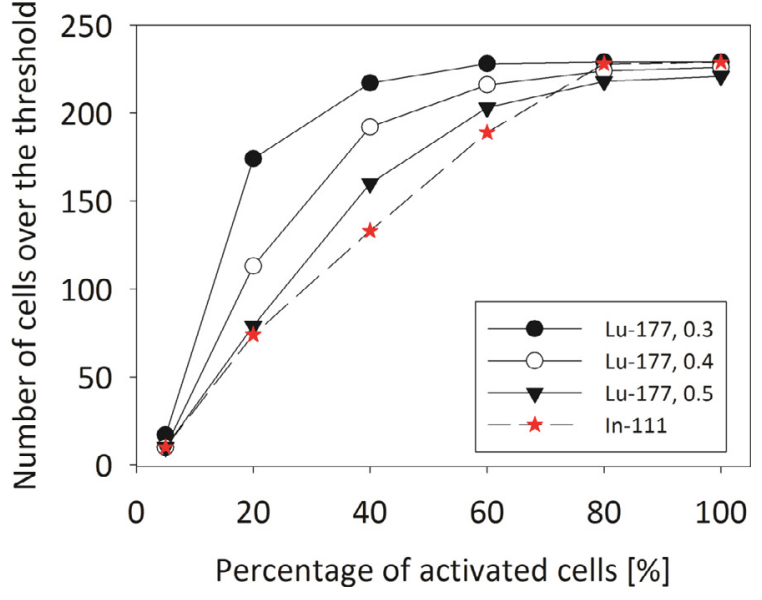

Fig. 6. The cross-fire effect demonstrated with ${ }^{177} \mathrm{Lu}$ vs. ${ }^{111}$ In cell distributions with a given dose threshold for cell death. A certain percentage of the cell nuclei (horizontal axis) in the spheroid is filled with an activity level that leads to doses higher or equal to the given lethal dose threshold in some of the cells of the spheroid (vertical axis). When the threshold value is increased, the curve for ${ }^{177} \mathrm{Lu}$ sinks as shown with black spheres (30\% of maximum dose), white spheres ( $40 \%$ of maximum dose), and black triangles (50\% of maximum dose). In this range, there is no noticeable change in the corresponding curves for ${ }^{111}$ In (red stars). (For interpretation of the references to colour in this figure legend, the reader is referred to the web version of this article.)

mented in studying radiopharmaceuticals for MRT (see e. g. $[8,9])$. In these studies, however, the activity has often been assumed to distribute homogeneously in the cells, and there is uncertainty of how to include the dose equivalent from radiopharmaceuticals to assessed endpoints such as spheroid control probability (SCP, the dose-dependent proportion of non-regrown spheroids after irradiation) and spheroid control dose $50 \%\left(\mathrm{SCD}_{50}\right.$, the irradiation dose inducing $50 \%$ loss of spheroid regrowth). We agree that spheroids can be utilized as efficient in vitro tools in drug development to study internalization, efficiency and metabolism of new radiopharmaceutical candidates, but in that case, dosimetry needs to be carried out carefully to interpret the results correctly.

Here, we demonstrated the importance of an appropriate scale for dosimetric considerations: if the range of the radiation is micrometers, the dosimetry also needs to be studied at that scale. At worst, heterogeneity in the activity distribution might cause MRT to fail, but as our examples show, there are many factors that affect the result: in particular, we exemplified the effect of the radionuclide ( ${ }^{111}$ In vs. ${ }^{177} \mathrm{Lu}$ ) and the degree of radiosensitivity of the tumor cells (three different, hypothetical thresholds for lethal cell dose) in the Hep2G spheroid geometry (Fig. 6). These results demonstrate the complexity of the optimization of MRT and how important it is to understand internal dosimetry. The effects of the temporal behavior of the radioactivity (dose rate, uptake and clearance) should also be studied. A pressing issue is to image the activity distribution with the needed accuracy in order to complete the workflow.

Both imaging and dosimetric methods are constantly gaining resolution, and the study of radiobiology can be taken to the next level accordingly. Automated segmentation algorithms are needed to process larger amounts of data quickly, and we are further improving our segmentation algorithm: the presented method is free of parameters excluding the width of the Gaussian filter during preprocessing and the definition of the structuring elements used in the morphological processing steps. Additionally, the generalization capability of our approach (applying the SVM classifier to other image datasets) should be validated. This way, the segmentation step for 3D image data should be easy to apply for the 
user in the future, in contrast to the manual segmentation process that can take hours of work. For the dose calculations we used a Monte Carlo method, which was time-consuming. More efficient computational approaches need to be studied at the scales relevant in MRT [29].

The established multicellular dosimetric chain can be further developed into a user-friendly platform so that the dose distribution in tumor and healthy tissues can be systematically studied in the case of different radiopharmaceuticals and, eventually, patients. This way, pre-clinical studies on new radiopharmaceuticals may be performed more efficiently, and, ultimately, a more predictive personalized administered activity-response relationship could be obtained for clinical practice.

\section{Conclusions}

We established a novel dose calculation software for 3D cell cultures and molecular radiotherapy. Our work shows that 3Dcultured tumor spheroids offer a promising tool for a radiopharmaceutical dosimetric scheme, as they provide biologically relevant data for dose calculations. The effect of activity uptake heterogeneity on the absorbed radiation dose distribution was evaluated in the case of different radionuclides $\left({ }^{125} \mathrm{I},{ }^{111} \mathrm{In}\right.$ and $\left.{ }^{177} \mathrm{Lu}\right)$ and the results demonstrate that radiation dosimetry is necessary in optimizing MRT. Our multicellular dosimetric chain has the potential to be widely applied to studying radiopharmaceuticals in different tissue models in order to gain new radiobiological data for drug development, nuclear medicine, and basic research.

\section{Acknowledgements}

L.K.K. acknowledges support from the doctoral programme in Materials Research and Nanosciences. Y.-R.L. acknowledges support from the Academy of Finland (No. 294193). M.Y. is thankful for the Orion Foundation for the funding of the professors' pool. We would like to thank the Light Microscopy Unit at the Institute of Biotechnology for technical guidance in confocal microscopy.

\section{References}

[1] Strigari L, Konijnenberg M, Chiesa C, Bardies M, Du Y, Gleisner KS, et al. The evidence base for the use of internal dosimetry in the clinical practice of molecular radiotherapy. Eur J Nucl Med Mol Imaging 2014;41:1976-88.

[2] McGowan DR, Guy MJ. Time to demand dosimetry for molecular radiotherapy? Br J Radiol 2015;88:20140720.

[3] Bolch WE, Bouchet LG, Robertson JS, Wessels BW, Siegel JA, Howell RW, et al. MIRD pamphlet No. 17: the dosimetry of nonuniform activity distributionsradionuclide S values at the voxel level. Medical Internal Radiation Dose Committee. J Nucl Med 1999;40:11S-36S.

[4] Siegel JA, Thomas SR, Stubbs JB, Stabin MG, Hays MT, Koral KF, et al. MIRD pamphlet no. 16: techniques for quantitative radiopharmaceutical biodistribution data acquisition and analysis for use in human radiation dose estimates. J Nucl Med 1999;40:37S-61S.

[5] Vaziri B, Wu H, Dhawan AP, Du P, Howell RW. SNMMI MIRD Committee. MIRD pamphlet No. 25: MIRDcell V2.0 software tool for dosimetric analysis of biologic response of multicellular populations. J Nucl Med 2014;55:1557-64.
[6] Zanzonico P. Cell-level dosimetry and biologic response modeling of heterogeneously distributed radionuclides: a step forward. J Nucl Med 2011;52:845-7.

[7] Stadler M, Walter S, Walzl A, Kramer N, Unger C, Scherzer M, et al. Increased complexity in carcinomas: analyzing and modeling the interaction of human cancer cells with their microenvironment. Semin Cancer Biol 2015;35:107-24.

[8] Ingargiola M, Runge R, Heldt JM, Freudenberg R, Steinbach J, Cordes N, et al. Potential of a Cetuximab-based radioimmunotherapy combined with external irradiation manifests in a 3-D cell assay. Int J Cancer 2014;135:968-80.

[9] Roa WH, Yaremko B, McEwan A, Amanie J, Yee D, Cho J, et al. Dosimetry study of [I-131] and [I-125]-meta-iodobenz guanidine in a simulating model for neuroblastoma metastasis. Technol Cancer Res Treat 2013:12:79-90.

[10] Valimaki JP, Lampinen JS, Kuronen AA, Ilvonen SA, Stepanek J, Savolainen SE. Comparison of different cell-cluster models for cell-level dosimetry. Acta Oncol 2001;40:92-7.

[11] Viehweger K, Barbaro L, Garcia KP, Joshi T, Geipel G, Steinbach J, et al. EGF receptor-targeting peptide conjugate incorporating a near-IR fluorescent dye and a novel 1,4,7-triazacyclononane-based (64)Cu(II) chelator assembled via click chemistry. Bioconjug Chem 2014;25:1011-22.

[12] Russell RA, Adams NM, Stephens DA, Batty E, Jensen K, Freemont PS. Segmentation of fluorescence microscopy images for quantitative analysis of cell nuclear architecture. Biophys J 2009;96:3379-89.

[13] Vincent L. Morphological grayscale reconstruction in image analysis: applications and efficient algorithms. IEEE Trans Image Process 1993;2:176-201.

[14] Cortes C, Vapnik V. Support-vector networks. JMRL 1995;20:273-97.

[15] Stabin MG, da Luz LC. Decay data for internal and external dose assessment. Health Phys 2002;83:471-5.

[16] Knowles BB, Howe CC, Aden DP. Human hepatocellular carcinoma cell lines secrete the major plasma proteins and hepatitis B surface antigen. Science 1980;209:497-9.

[17] Bhattacharya M, Malinen MM, Lauren P, Lou YR, Kuisma SW, Kanninen L, et al Nanofibrillar cellulose hydrogel promotes three-dimensional liver cell culture. J Control Release 2012;164:291-8.

[18] Malinen MM, Kanninen LK, Corlu A, Isoniemi HM, Lou YR, Yliperttula ML, et al. Differentiation of liver progenitor cell line to functional organotypic cultures in 3D nanofibrillar cellulose and hyaluronan-gelatin hydrogels. Biomaterials 2014;35:5110-21.

[19] Baró J, Sempau J, Fernàndez-Varea JM, Salvat F. PENELOPE: an algorithm for Monte Carlo simulation of the penetration and energy loss of electrons and positrons in matter. Nucl Instrum Methods Phys Res 1995;100:31-46.

[20] Papadimitroulas P, Loudos G, Nikiforidis GC, Kagadis GC. A dose point kernel database using GATE Monte Carlo simulation toolkit for nuclear medicine applications: comparison with other Monte Carlo codes. Med Phys 2012;39:5238-47.

[21] Hippelainen E, Tenhunen M, Sohlberg A. Fast voxel-level dosimetry for (177)Lu labelled peptide treatments. Phys Med Biol 2015;60:6685-700.

[22] Fedorov A, Beichel R, Kalpathy-Cramer J, Finet J, Fillion-Robin JC, Pujol S, et al. 3D slicer as an image computing platform for the quantitative imaging network. Magn Reson Imaging 2012;30:1323-41.

[23] Taha AA, Hanbury A. Metrics for evaluating 3D medical image segmentation: analysis, selection, and tool. BMC Med Imaging 2015;15. 29,015-0068-x.

[24] Maska M, Ulman V, Svoboda D, Matula P, Matula P, Ederra C, et al. A benchmark for comparison of cell tracking algorithms. Bioinformatics 2014;30:1609-17.

[25] Otsu N. A threshold selection method from gray-level histograms. IEEE Trans Syst Man Cybern 1979;9:62-6.

[26] Bodei L, Kassis AI, Adelstein S], Mariani G. Radionuclide therapy with iodine125 and other auger-electron-emitting radionuclides: experimental models and clinical applications. Cancer Biother Radiopharm 2003;18:861-77.

[27] Lind BK, Brahme A. The radiation response of heterogeneous tumors. Phys Med 2007;23:91-9.

[28] Malaroda A, Flux GD, Buffa FM, Ott RJ. Multicellular dosimetry in voxel geometry for targeted radionuclide therapy. Cancer Biother Radiopharm 2003;18:451-61.

[29] Traino AC, Piccinno M, Avigo C. Dosimetry of non-uniform activity distribution: possibility to use the local energy deposition approach at the voxel level in radionuclide therapy. Biomed Phys Eng Expr 2016;2(6). 